\title{
Policy Implications of Downscaling the Time Dimension in Power System Planning Models to Represent Variability in Renewable Output
}

\author{
Lina Reichenberg \\ Department of Energy and Environment, Chalmers University of Technology, Sweden \\ Afzal S. Siddiqui* \\ Department of Statistical Science, University College London, United Kingdom, Department of Computer \\ and Systems Sciences, Stockholm University, Sweden, and Department of Decision Sciences, HEC \\ Montréal, Canada \\ Sonja Wogrin \\ Instituto de Investigación Tecnológica, Universidad Pontificia Comillas, Spain
}

\begin{abstract}
Due to computational constraints, power system planning models are typically unable to incorporate full annual temporal resolution. In order to represent the increased variability induced by large amounts of variable renewable energy sources, two methods are investigated to reduce the time dimension: the integral approach (using typical hours based on demand and renewable output) and the representative days method (using typical days to capture annual variability). These two approaches are tested with a benchmark implementation that incorporates full time representation in order identify their suitability for assessing power systems with high renewable penetration. The integral method predicts renewable capacities within a 10\% error margin, this paper's main performance metric, using just 32 time steps, while the representative days approach needs 160-200 time steps before providing similarly accurate renewable capacity estimates. Since the integral method generally cannot handle variation management, such as trade and storage, without enhancing the state-space
\end{abstract}

\footnotetext{
${ }^{*}$ Corresponding author

Email address: afzal.siddiqui@ucl.ac.uk (Afzal S. Siddiqui*)
} 
representation, it may be more applicable to one-node models, while the representative days method is suitable for multi-regional models. In order to assess power systems with increasing renewable policy targets, models should be designed to handle at least the 160 time steps needed to provide results that do not systematically overestimate the renewable capacity share.

Keywords: Variable renewable energy sources, Power system planning models, Time slicing, State-space representation

\section{Introduction}

In most industrialised countries, concerns about climate change have prompted policymakers to mandate targets for increasing the share of variable renewable energy sources (VRES) such as solar and wind power (European Commission, 2011). In turn, policy-enabling models are relied upon to delineate the design of and transition into a future power system with less $\mathrm{CO}_{2}$ emissions. For example, ReEDS (Short et al., 2011) is a linear programming model for the U.S. that uses a demand-based integral method, i.e., representation of time by averages based only on demand fluctuations, with 17 time periods per year to capture variability. By contrast, the POWER model for the U.S. uses extreme days comprising variability in VRES output as well as demand with hourly resolution to study both flexibility (Frew et al., 2016) and storage (Frew and Jacobsen, 2016). LIMES-EU uses clustering techniques to select representative days in order to assess a sustainable transition for Europe (Nahmmacher et al., 2016). ${ }^{1}$

Such power system planning models are computationally demanding due to their technological and/or spatial detail. They are, therefore, restricted as to their representation of time, and they, thus, typically reduce the number of time steps to 10-20, e.g., 17 annual time steps in the integral model ReEDS and 4 representative days each in LIMES-EU and POWER. When these models originated, the main variable quantity was the demand, which

\footnotetext{
${ }^{1}$ The full technical documentation is available in Nahmmacher et al. (2014).
} 
fluctuates rather regularly depending on the time of day, the day of the week, and the season. Thus, variation may be adequately represented using 10 time steps. Although such a time representation does not capture variability on the generation side, viz., for solar and wind power, as long as the solar and wind contribution is marginal, the lack of linkage to variations in solar and wind output does not gravely impact model results. However, as the levelised cost of solar and wind generation has decreased rapidly to reach near parity with that of gas-fired plants (Energy Information Administration, 2017), VRES may potentially constitute a large part of the generation in a least-cost system. Consequently, it is desirable for policy-enabling planning models to represent variability in solar and wind power output. If a time representation based on demand variation were used in a scenario with low costs of variable generation, then the variations on the generation side will be underestimated, thereby leading to a gross overestimation of the optimal VRES capacity (Poncelet et al., 2016). Even models with more detailed temporal representation, e.g., using 8760 annual time steps, face a tradeoff in terms of less spatial resolution and more computational effort (MacDonald et al., 2016). Meanwhile, models with more engineering details, e.g., EnergyPLAN, calculate resulting power system operations once VRES capacities are known based on samples from statistical distributions. Yet, as indicated in the documentation (Lund and Thellufsen, 2017), EnergyPLAN "optimises the operation of a given energy system on the basis of inputs and outputs defined by the user." Unlike EnergyPLAN, the capacities are endogenously determined by the class of power system models that are studied in the current paper.

Recently, several articles have been produced highlighting the importance of a representation of time that is apt for power systems that can be expected to incorporate substantial levels of VRES as summarised by Pfenninger et al. (2014). In such studies, one may differentiate between two main families of methods to represent the variability in production introduced by VRES. One family of methods classifies the time into "typical" hours in terms of wind output and demand and then averages quantities within these classes. This family of 
time-reduction methods will be referred to as integral following Nahmmacher et al. (2016). This approach was developed for one-node models by Wogrin et al. (2014) with Lehtveer et al. (2016) implementing an application to a global model. Wogrin et al. (2016) further develop the approach to include storage using a transition matrix to relate the time slices to each other. The second type of method is to select so-called representative days, i.e., to choose certain days and assign unequal weights to them in order to replicate variation during the year with fewer time steps. Nahmmacher et al. (2016) develop a method to select representative days based on looking at each day's output of variable quantities (wind, solar, demand) as a vector. Frew and Jacobsen (2016) use a method where extreme days, e.g., the day with the highest demand and the lowest wind production, are included as well as random days. Merrick (2016) uses distance measures to determine how many representative days or weeks are sufficient to capture the effects of variability.

In terms of the number of time steps necessary to represent variability adequately, Wogrin et al. (2014) and Wogrin et al. (2016) evaluate the number of time slices needed to come within a certain range of the capacities obtained using a model with hourly resolution for a full year. They find that around 100 time slices plus the transition matrix to represent storage are needed for their unit-commitment model. ${ }^{2}$ Furthermore, they find that the CPU time is reduced to about a third compared to the hourly model of 8760 time steps. Nahmmacher et al. (2016) implement their LIMES-EU model with successively more representative days and find that 20-30 representative days (which amounts to 160-240 time steps) are necessary to converge to the correct system capacity mix. Frew and Jacobsen (2016) find that the system cost in their POWER model differs by less than $10 \%$ for 14 representative days as compared to a "full" implementation.

Besides the aforementioned power system planning models, there are others that either account for the interaction with other parts of the energy system or have a more detailed representation of strategic behaviour. For example, inadequate representation of variability

\footnotetext{
${ }^{2}$ This number could vary depending on the nature of the system and variability of the data.
} 
in power system modelling will have consequences for a holistic approach to smart energy systems (Lund et al., 2017). Likewise, the coupling of the power system with district heating in terms of integrating VRES is important for the Nordic region (Virasjoki et al., 2018). More generally, electricity and gas networks may also require further joint analysis (Zhao et al., 2018). Indeed, more adoption of VRES requires countervailing flexibility, which could be provided by heat storage, combined heat and power, and gas-fired power plants. Thus, additional VRES capacity could also affect the leverage of flexible generators in exerting market power, which would require a game-theoretic framework for analysis (Conejo et al., 2016).

Focusing on power system planning models, although the performance of integral and representative days methods has been evaluated individually against benchmark model results with a high time resolution, e.g., Frew and Jacobsen (2016), Nahmmacher et al. (2016), Wogrin et al. (2014), Wogrin et al. (2016), and Merrick (2016), the two approaches have not been compared with each other in the same model apart from an assessment with an equal number of time periods in Poncelet et al. (2016). In this paper, the performances of the two families of methods (integral and representative days) are compared in a costminimising power system planning model and evaluated with respect to how well they predict the capacity mix and the system cost.

The research questions addressed in this paper are:

- Which method comes closer to predicting the benchmark model ${ }^{3}$ VRES capacity (the main performance metric in this paper) for a given number (10-20) of time steps?

- Which method is more accurate if a larger number of time steps (200) is tractable?

- What, if any, is the impact of using a clustering method rather than arbitrarily picked days?

\footnotetext{
${ }^{3} \mathrm{~A}$ model with evaluation of a full year (2920 time steps) of data. This number of time steps corresponds to the availability of finer spatial resolution data from the European Centre for Medium-Range Weather Forecasts (ECMWF).
} 
From a policy perspective, answers to these questions are essential in assessing the impact of proposed environmental regulations on the power system. Since a conventional loadslicing approach in large-scale power system models leads to overestimates of VRES installed capacity, it would provide a biased basis on which to establish future energy policy. Hence, by directly comparing two enhanced time-resolution methods, this paper demonstrates how models should represent temporal dependence between VRES and demand in order to provide a credible basis for policymaking.

\section{Methods}

The two time-reduction methods (integral and representative days) are tested using data for several European regions: one each in Germany, Denmark, Spain, France, and Ireland. The regions are chosen to represent a variety in terms of VRES and demand conditions. Demand and weather data are the input to a power system planning model. The results of the time-reduction methods are compared with those from a fully time resolved linear optimisation model using 2920 time steps (from now on called the benchmark model). The measure of accuracy for the two approaches and the main performance metric used in this paper is their ability to predict the optimal capacity investment for the different technologies, especially VRES, using results from the benchmark model as comparison.

The two methods are described in steps and illustrated with an example. Several preparation methods have been proposed in order to group data into representative periods, e.g., via either hierarchical (as in Nahmmacher et al. (2016) and Merrick (2016)) or k-means clustering (as in Frew and Jacobsen (2016) and Wogrin et al. (2014)). The latter technique partitions n demand and VRES pairs into $k$ clusters via an iterative procedure that minimises the sum of weighted distances between each pair and cluster centroid. By contrast, hierarchical clustering starts with clusters of size one data point each and sequentially merges nearby clusters. ${ }^{4}$

\footnotetext{
${ }^{4} \mathrm{k}$-means clustering is implemented using the built-in MATLAB command kmeans with the option 'replicates.' k-mediods may also be used to represent the "true" representative point for a system state. The results for the representative days approach are similar whether hierarchical or k-means clustering
} 
Since k-means clustering is used here, which depends on initial points, 200 replicates are performed and the closest match is picked. The data are normalised so that solar, wind, and demand observations all attain values between 0 and 1 (see Section 3.1).

\subsection{Representative Days}

Representative days are selected as described in Nahmmacher et al. (2016) ${ }^{5}$ except for the clustering method: in Nahmmacher et al. (2016) hierarchical clustering is used, while $\mathrm{k}$-means clustering is used here. The procedure is illustrated in Figure 1a and is as follows:

- Partition the data into vectors each consisting of the output of each variable quantity (in this case, solar, wind, and demand) during one day.

- Cluster the vectors into the chosen number of clusters using k-means clustering.

- Pick the day(s) that are closest to the centroids as representative. The number of days in a cluster is used as the weight for that representative day.

- Run the model with the time steps pertaining to these days.

Since there are $n$ representative days with time resolution of three hours per step, i.e., $8 n$ time steps are selected, this amounts to:

- Partition the data into 365 vectors, each with 24 elements (elements 1:8 are the wind output, elements 9:16 are the solar output, elements 17:24 are the demand).

- Cluster the vectors into $n$ clusters using k-means clustering.

- Pick the $n$ days that are closest to the centroids as representative. The number of days in a cluster is used as the weight for that representative day. An example of a time series resulting from this procedure can be found in Figure 2a.

is used.

${ }^{5}$ This method is chosen despite the fact that the inclusion of extreme days, as in Frew and Jacobsen (2016), seems to yield better results. The justification for this approach is to be fair to the two investigated families of methods, and it is not obvious how the equivalent of selecting extreme events would be performed for the integral method. 
- Run the model with the $8 n$ time steps pertaining to these days.

The number of representative days, $n$, is between 1 and 25, which leads to between 8 and 200 time steps (Table 1). Finally, to evaluate the performance of the k-means clustering for selecting representative days, the approach of picking random days is also attempted here.

Table 1: Attributes of data used in this analysis

\begin{tabular}{|c|c|}
\hline Time resolution & 3 -hourly \\
Time steps per year & 2920 \\
Time steps per day & 8 \\
Varying quantities & 3 (demand, wind, solar) \\
Number of time steps in modelling & $8-200$ (equivalent of $1-25$ days) \\
\hline
\end{tabular}

\subsection{Integral}

The principle behind integral methods is to identify states. States may be described as "typical" situations, e.g., when the wind power output is low and the demand is high. This is illustrated in Figure 1b, which shows an example where states are based on wind power output and demand only. The method to identify states in this paper follows Wogrin et al. (2014):

- Partition the data into vectors each consisting of the outputs of variable quantities for one time step. Each such vector is called a state as in Wogrin et al. (2014) and Wogrin et al. (2016).

- Cluster the vectors into the chosen number of states using k-means clustering.

- Take the centroid of each cluster output for that state. The number of elements in a cluster is used as the weight for that state in the modelling.

- Run the model using the states and weights.

In this case, this amounts to:

- Partition the data into vectors with three elements each: solar, wind, and demand for one time step. There are 2920 such vectors. Each such vector is a state. 
- Cluster the vectors into $n^{\prime}$ clusters using k-means clustering.

- Take the centroid of each cluster, i.e., a triplet with values for solar, wind, and demand, and use it as the solar output/wind output/demand for that time step. The number of elements in a cluster is used as the weight for that time step. An example of a time series resulting from this procedure can be found in Figure $2 \mathrm{~b}$.

- Run the model using the time steps and weights.

The number of states, $n^{\prime}$, is between 8 and 200, in steps of 8 (Table 1).

As a basis for comparison in terms of predicting the VRES capacity and system cost accurately (although analysis with other metrics, e.g., VRES curtailment, may also be implemented as in the Appendix), a demand-based integral method is adopted, similar to that in Short et al. (2011) and Odenberger et al. (2009), for example. The procedure to find the demand-based states is exactly like the integral method (above), but the states are based only on demand. Consequently, solar and wind conditions do not influence the selection of time steps that define a state in such a conventional representation of time.

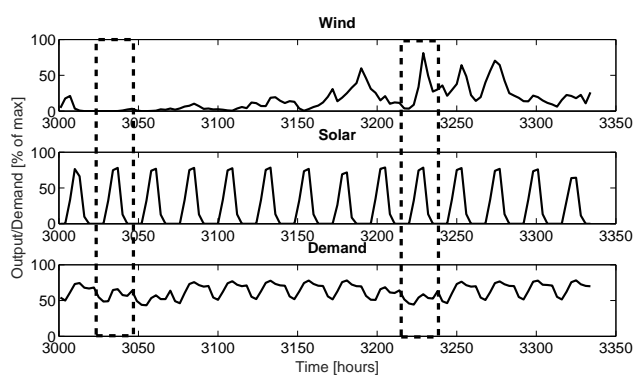

(a) Representative days to capture the variation during the entire year

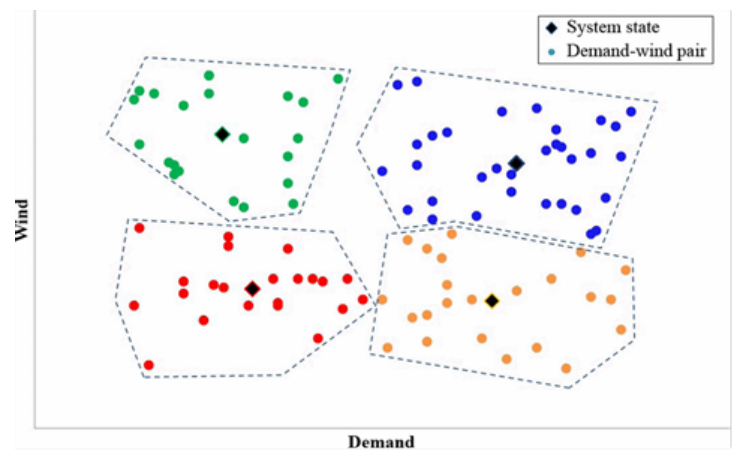

(b) Integral approach based on partitioning variable quantities by their means (schematic based on only wind power output and demand)

Figure 1: Time-reduction methods 

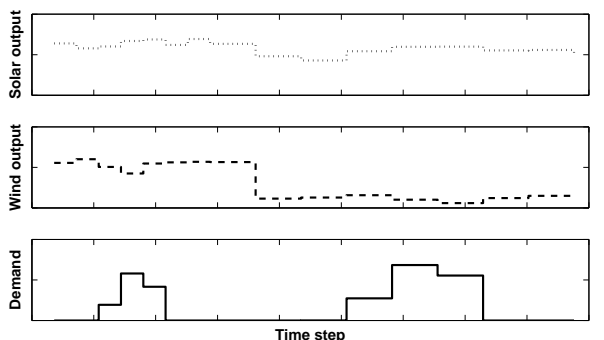

(a) Representative days
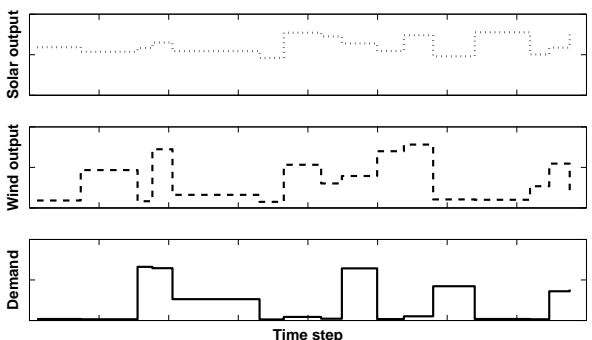

(b) Integral approach

Figure 2: Time series produced with 16 time steps based on k-means clustering

\subsection{Optimisation Model}

The basis for comparing the representative days and the integral time-resolution methods is a one-node cost-minimisation linear programming model. ${ }^{6}$ Demand is assumed to be perfectly price inelastic but varying in time. The model's decision variables are (i) investment capacities in a number of technologies (set $\mathcal{I}$ ) and (ii) their production over representative time periods (set $\mathcal{T}$ ) in order to minimise annualised investment and operating costs for a target year. The only constraints are that (i) total generation should meet demand at every time step and (ii) output is limited by available capacity. For the VRES technologies, operating costs are zero, but availability depends on weather-based factors. The model is run with different weights on the time steps $($ set $\mathcal{T}$ ) corresponding to the cluster sizes. For the benchmark model, all 2920 time steps have the same weight.

\subsubsection{Sets}

$\mathcal{I}$ Technologies available for investment.

$\mathcal{T}$ Time steps/slices.

\subsubsection{Parameters}

$c_{i}$ Annualised investment cost for technology $i \in \mathcal{I}(€ / \mathrm{MW})$.

\footnotetext{
${ }^{6}$ The comparison of the two time-resolution methods may depend on the type of model and constraints. For example, Wogrin et al. (2016) use a unit-commitment model with binary decision variables, while TejadaArango et al. (2017) have network constraints.
} 
$\ell_{t}$ Demand at time step $t \in \mathcal{T}(\mathrm{MWh})$.

$r_{i}$ Operating cost for technology $i \in \mathcal{I}(€ / M W h)$.

$v_{t}$ Weight on time step $t \in \mathcal{T}(\mathrm{h})$.

$w_{i t} \in[0,1]$ Availability factor for technology $i \in \mathcal{I}$ during time step $t \in \mathcal{T}(-)$.

\subsubsection{Decision Variables}

$C_{i}$ Capacity investment in technology $i \in \mathcal{I}(\mathrm{MW})$.

$P_{i t}$ Production from technology $i \in \mathcal{I}$ during time step $t \in \mathcal{T}(\mathrm{MWh})$.

\subsubsection{Constraints}

\section{Energy balance}

Total production must meet demand during each time step:

$$
\sum_{i \in \mathcal{I}} P_{i t} \geq \ell_{t}, \forall t \in \mathcal{T}
$$

\section{Production constraint}

Production by a given technology cannot exceed available capacity during each time step:

$$
P_{i t} \leq v_{t} w_{i t} C_{i}, \forall i \in \mathcal{I}, \forall t \in \mathcal{T}
$$

\subsubsection{Objective Function}

The objective function is to minimise annualised cost of investment and operations:

$$
\min _{C_{i} \geq 0, P_{i t} \geq 0} \sum_{i \in \mathcal{I}} \sum_{t \in \mathcal{T}} r_{i} P_{i t}+\sum_{i \in \mathcal{I}} c_{i} C_{i}
$$

subject to constraints (1)-(2). 


\section{Numerical Examples}

\subsection{Data}

The data consist of time series for demand, solar, and wind for five regions: one each in Denmark, Germany, France, and Spain, as well as the entire island of Ireland. The investment and operating costs for energy technologies are listed in Table 2. The demand data are available on an hourly basis from Eurostat (European Commission, 2015). Since the weather data are available only for every three hours, the demand data points are taken for the same hours, i.e., 2920 time steps for one year. ${ }^{7}$ The demand data are used as they are in the optimisation step. However, for the preparation step (integral or representative days method), the demand data are normalised so that the maximum demand event is given a value of 1 . The wind and solar data input is based on weather data from the European Centre for Medium-Range Weather Forecasts (ECMWF), processed through a turbine function and a function to mimic the output of solar PV (see Reichenberg et al. (2017) for a more thorough description). Solar and wind output also attain values between 0 and 1 , but 1 is equivalent to the nameplate capacity. Sample code and a generic dataset for executing the procedure are available as an electronic companion to this paper.

In addition to the investment options of solar and wind technologies, there are four thermal technology options in the set $\mathcal{I}$, defined by their annualised investment costs and operating costs based on technology data forecasts from Energy Information Administration (2017) and Fraunhofer Institute for Solar Energy Systems (2013) to allow for sufficient VRES and dispatchable energy capacity to permit an assessment of the time-resolution methods (Table 2). The interest rate is set to $5 \% /$ annum, and investment costs are annualised over the technical lifetimes of the plants. ${ }^{8}$

\footnotetext{
${ }^{7}$ Alternatively, it would have been possible to have used 8760 time steps with the same weather data replicated for every three successive time steps. However, since the paper's objective is to compare models that downscale time representation with a benchmark model that uses the entire available temporal resolution, using the given dataset is not a limitation.

${ }^{8}$ In Table 2, the entry in the final column corresponds directly to each technology's $r_{i}$ parameter. The $c_{i}$ parameter is calculated using the annuity formula, e.g., $c_{\text {gas }}=40+\frac{0.05 \times 1000000}{1-1.05^{-40}}$ or $€ 58318 / \mathrm{MW}$.
} 
Table 2: Technology attributes

\begin{tabular}{|c|c|c|c|c|}
\hline Technology & $\begin{array}{c}\text { Investment Cost } \\
(\mathrm{k} € / \mathrm{MW})\end{array}$ & $\begin{array}{c}\text { Annual Fixed } \\
\text { Cost }(\mathrm{k} \in / \mathrm{MW})\end{array}$ & $\begin{array}{c}\text { Lifetime } \\
(\mathrm{a})\end{array}$ & $\begin{array}{c}\text { Operating Cost } \\
(€ / \mathrm{MWh})\end{array}$ \\
\hline Wind & 700 & 0 & 25 & 0 \\
Solar & 480 & 0 & 25 & 0 \\
Nuclear & 2500 & 43 & 60 & 18 \\
Coal & 1700 & 43 & 40 & 45 \\
Gas & 1000 & 40 & 40 & 52 \\
Backstop & 100 & 0 & 40 & 120 \\
\hline
\end{tabular}

\subsection{Results}

The approach of Nahmmacher et al. (2016) is followed here in using the VRES capacity and the system cost (the value of the objective function) to measure accuracy. The additional measure of the generation per technology is used by Wogrin et al. (2014), Frew and Jacobsen (2016), and Merrick (2016). For application to investment models, however, the capacity mix is vital, while the dispatch is not since, given the correct capacities, the dispatch can be determined using a dispatch model in a post-investment analysis. Out of the technologies, the VRES capacity sets the scene for the remaining generation, and, therefore, the exposition of the results is concentrated mainly on these.

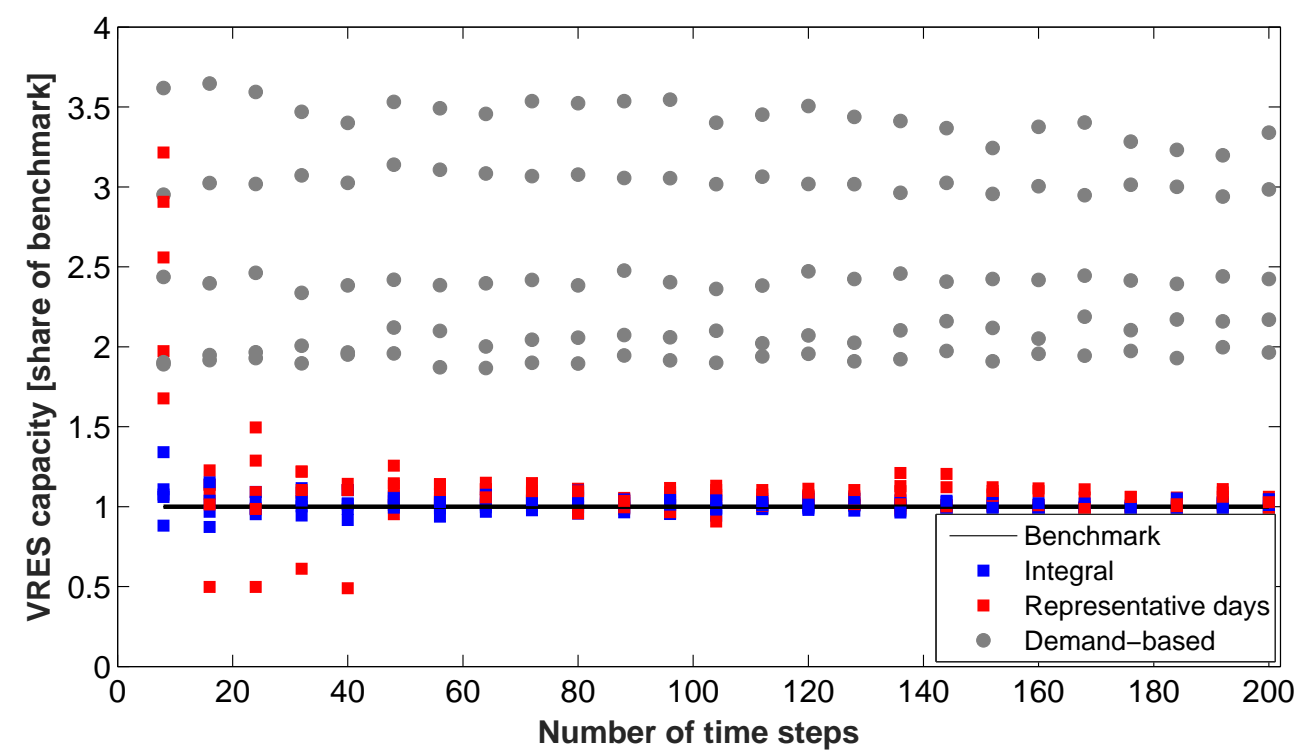

Figure 3: Performance of time-reduction methods in predicting the VRES capacity 


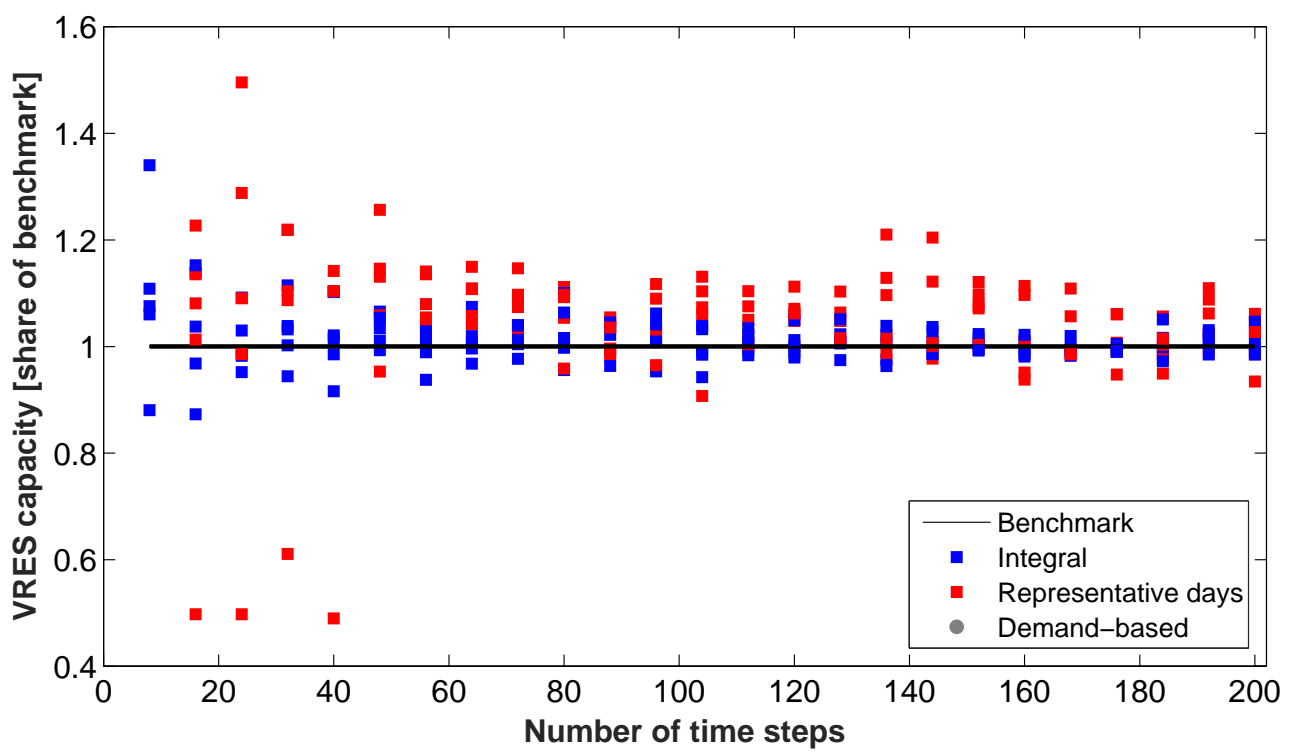

Figure 4: Performance of time-reduction methods in predicting the VRES capacity (zoom-in of Figure 3)

\subsubsection{Accuracy in Computing Optimal Capacity Mix and System Cost}

Figure 3 shows the deviation of the integral demand-based, integral, and representative days approaches from the VRES capacity predicted, which is the main performance metric used here, by the benchmark model for all regions. The benchmark results are those from a model run with an entire year of data 2920 time steps. The vertical axis measures the VRES capacity relative to that predicted by the benchmark model, and each dot represents one of the five regions.

In order to establish the necessity of replacing the use of demand-based states in models with a different method for time representation, Figure 3 shows the performance of the demand-based slicing in gray. It is clear that, regardless of how many time steps in the modelling (=states) that are allowed, this method overestimates the optimal amount of VRES capacity by a factor of 2-4. Thus, neglecting VRES variation in time slicing and relying solely on the demand to downscale the time dimension will lead to significant inaccuracies from the perspective of capturing VRES capacity.

If the time representation is to be used in a model that allows for only 16-40 time steps, then the integral method gives a much better prediction of the VRES capacity: the regions 
with maximum discrepancy are 5-15\% off the correct capacity compared to the representative days method, where the maximum discrepancy is $40-50 \%$ off the correct capacity (Figures 3and 4). The integral approach is remarkably stable and within a 10\% error of the benchmark VRES capacity for all runs with more than 32 time steps. The representative days approach may miscalculate the optimal VRES capacity by $50 \%$ for less than 40 time steps. It is rare that the representative days approach miscalculates VRES capacity by more than $20 \%$ for a number of time steps of 160 (20 days) or more (Figure 3). This is in line with the results for the test with the more detailed models in references Nahmmacher et al. (2016) and Frew and Jacobsen (2016).

The system cost (Figure 5) shows less discrepancy between the results with fewer time steps and the benchmark model results compared to the VRES capacity (Figure 3). However, the system cost output from an investment model hides discrepancies in capacity mix that would have a greater impact on cost had they been evaluated in, e.g., a unit-commitment model. The system cost is underestimated for low numbers of time steps, especially for the case of integral demand-based slicing (Figure 5), while the VRES capacity is overestimated (Figure 3). When the variability of VRES to a large extent is averaged out (such as is the case for demand-based slicing), the load-covering capacity of VRES is overestimated, and, thus, the optimal capacity of VRES is also overestimated. This leads to an underestimate of system cost. Hence, the demand-based slicing approach also underperforms on the basis of the system cost as a metric.

Both methods (integral and representative days) also predict the thermal capacities fairly well. However, one exception is backstop capacity, the need for which depends heavily on extreme events (high demand, low wind, low solar) (Figure 6). Although results for only the Danish region are shown, similar trends are observed for other regions, viz., an underestimate of peak capacity by both reduction methods. 


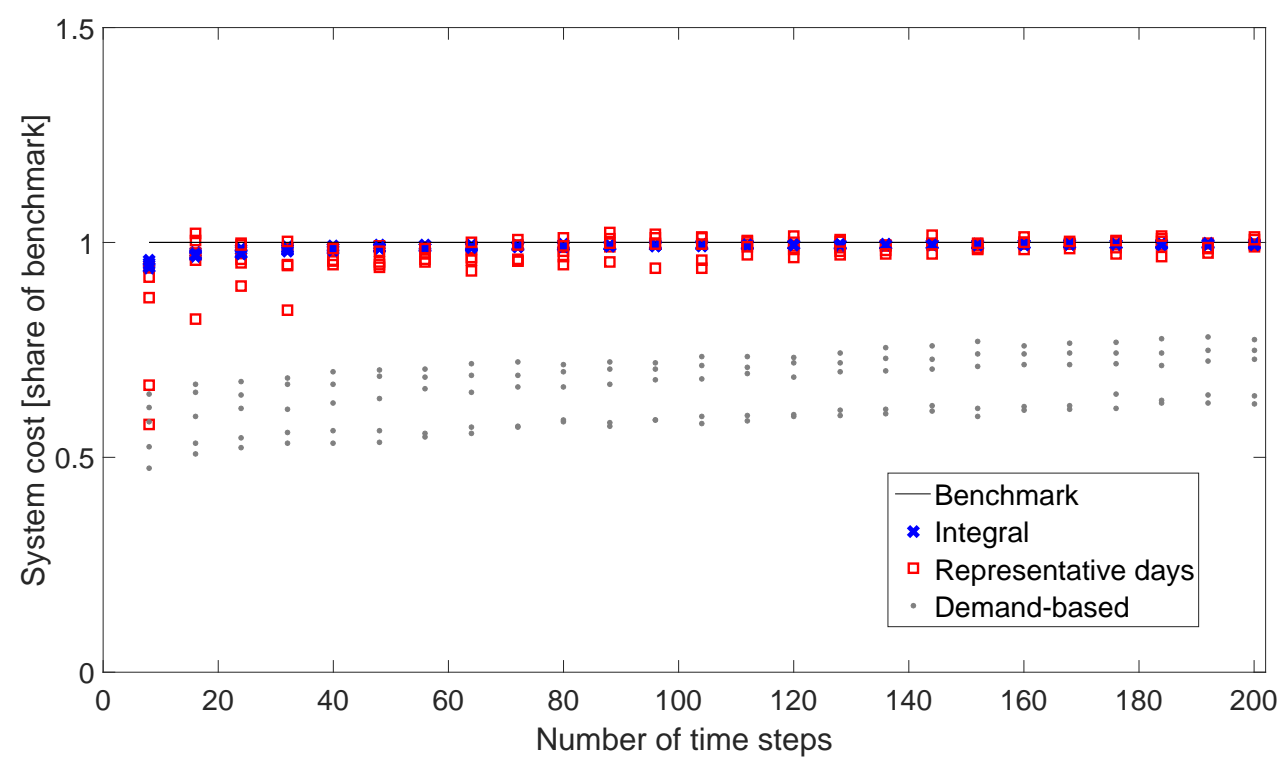

Figure 5: Performance of time-reduction methods in predicting the system cost

\subsubsection{Performance of Randomly Selected Days}

As a corollary to the results on the two methods, results are presented on the importance of the preparation method. Figure 7 shows the alternative of picking random days instead of performing the preparation step of finding the representative days. As a result, the randomdays method may be off by $50 \%$ of the VRES capacity predicted by the benchmark model for as many as 168 time steps (21 days) ${ }^{9}$. Overall, the variance of the VRES capacity is much higher for the random days than for representative days picked using k-means clustering. A similar result is obtained for the integral methods when intervals for finding states are arbitrarily chosen instead of using the clustering method.

\section{Discussion}

Two methods (integral and representative days) to downscale the time dimension in power system planning models are compared in order to represent better the cases with a high share of variable generation. The comparison is performed in a simple model, where benchmark

\footnotetext{
${ }^{9}$ In fact, when extending the number of randomly picked days beyond the 25 shown here, the occasionally large errors persisted even at 100 days (800 time steps).
} 


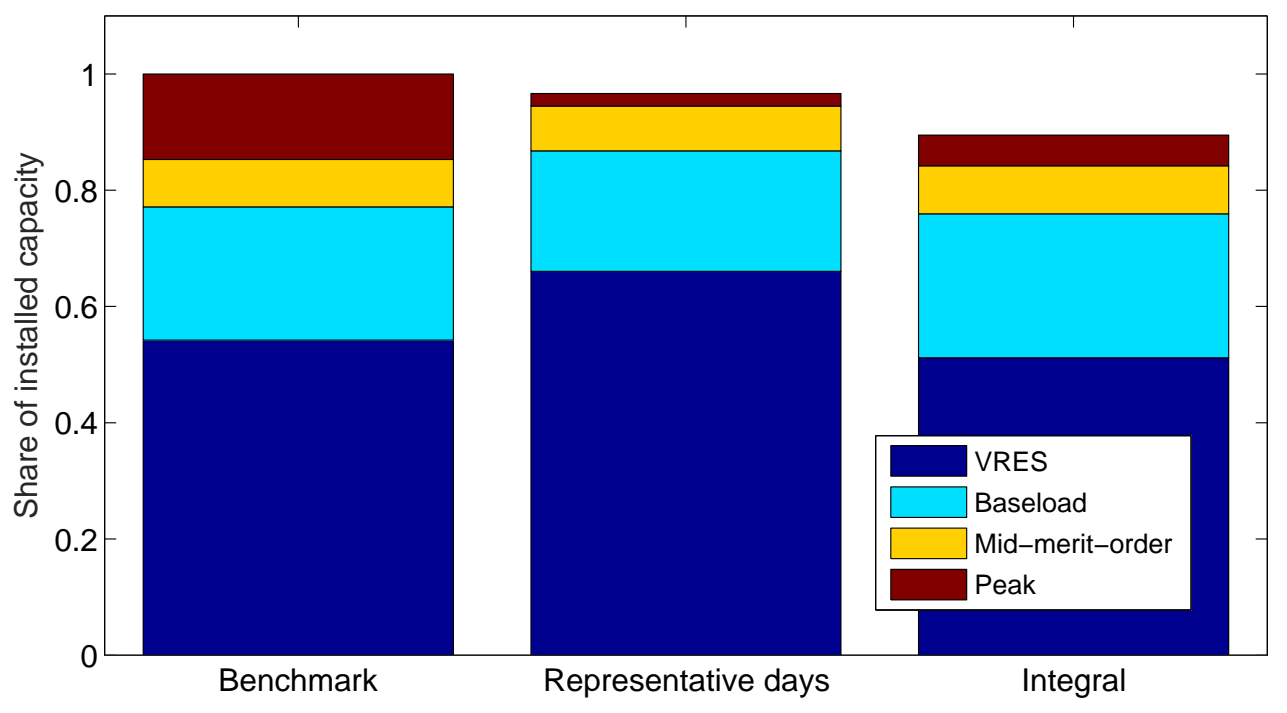

Figure 6: Installed capacities for the case with 32 time steps (Danish region)

results obtained with the full time resolution may be used as a comparison. The number of time steps to predict VRES capacity within a $10 \%$ error margin is approximately 30 for the integral method and approximately 160 for the representative days method. This result may inform developers in choosing an appropriate time representation method for their model and design the model so that it is tractable with the number of time steps needed to come within an acceptable error margin.

Of the two methods tested here, the integral approach has a smaller standard deviation of the resulting VRES capacity for different runs of the heuristic to pick the states/representative days. Even for a low number of time steps, e.g., 32, the integral method with k-means clustering is within 10\% accuracy in predicting the VRES capacity. Wogrin et al. (2016) use a more complex (unit-commitment) model and find that around $10^{2}$ time slices are needed. The representative days approach in this work needs 160-200 time steps to reach a $10 \%$ accuracy level. The value for the representative days approach in the present study is similar to the results in Nahmmacher et al. (2016) and Frew and Jacobsen (2016). Frew and Jacobsen (2016) use another preparation method to find the representative days, which leads to an overestimate of the system cost for around $10^{2}$ time steps, rather than, as here and in 


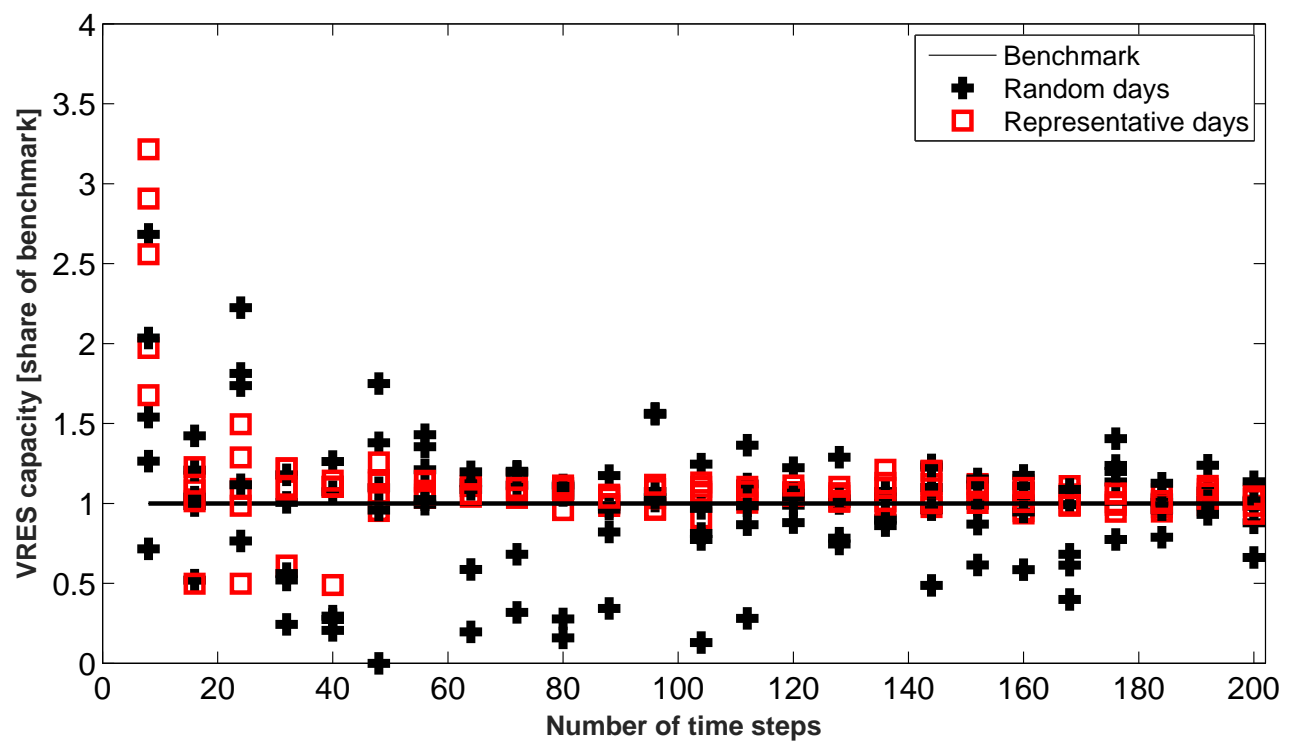

Figure 7: Performance of randomly selected days in predicting the system cost

Nahmmacher et al. (2016), an underestimate of the system cost. Merrick (2016) uses more stringent measures of accuracy: since he requires several measures (e.g., annual generation) to be within a range of the correct value than just the VRES capacity, his estimate of the number of necessary time steps is instead on the order of $10^{3}$. Thus, it seems likely that at least 160 time steps are necessary to represent variability with the representative days approach. Due to intractability, many power system planning models cannot use as many as 160 time steps, and, thus, in order to assure a reliable result, they would need to be redesigned, in effect reducing the technical and/or spatial complexity of the models.

The model that is used for comparing the two time-reduction methods here is a (i) simple (ii) one-node model that (iii) lacks storage options. These three limitations entail that the present experiment can cover only representation of variability and not all variation management strategies. ${ }^{10}$ Variation management includes (i) flexibility of the surrounding (mainly thermal) generation system, (ii) flexibility of demand (such as demand-side management, DSM), (iii) curtailment of VRES electricity, (iv) variability smoothing by transmission invest-

\footnotetext{
${ }^{10}$ Except for curtailment.
} 
ment and trade, and (v) storage. These measures become more important as the penetration level of variables increases, and, thus, for many applications, these are, indeed, important to incorporate into models and the method to downscale time. The testing framework is necessarily simplified by using only the technologies that both types of representation methods can incorporate. For example, the representative days method can handle trade and short-term storage but not overnight storage or hydropower, whereas the integral method has traditionally not been able to handle spatial aspects of a power network. Hence, only by boiling the testing framework down to the current one can one meaningfully compare the two methods' performance in predicting VRES capacity shares directly.

Of the two methods tested here, the representative days method can incorporate trade since it may be employed for network models as is done in Frew et al. (2016), Nahmmacher et al. (2016), and Haller et al. (2012). The representative days method can also represent storage and DSM that takes place within the day-night, which may be a sufficiently good representation for storage that is used mainly for solar power. However, it can neither handle storage over longer periods nor represent the dynamics of hydropower, which may be viewed as a type of storage. The integral method, on the other hand, has traditionally not been employed in a network model with many nodes. Only recently has the integral method been adapted to handle storage operations (but not investment) in a congested network (Tejada-Arango et al., 2017). Thus, it needs further enhancement to tackle investment in the potentially important variation management tool of transmission investment and trade. Both approaches tested here can be expanded to include more variation management strategies. The integral method may be complemented with a transition matrix, as shown in Wogrin et al. (2014), in order to cover cycling and storage. The representative days approach may be expanded to choose representative weeks instead of days, which would a priori be more suited to represent long-term storage, cycling, and hydropower. Both expansions make models larger, thereby increasing computation time, which may render problem instances intractable. A study that extends the test ground for the two families of methods to one that can incorporate all 
variation management strategies would be beneficial to both the modelling community and policymakers.

The results comparing random days with those selected using one of the two methods show that the preparation step (in this case k-means clustering) is important and impacts the tendency of the model results with the reduced time dimension to underestimate or overestimate results. In this paper, random picking of days provides little certainty about the accuracy of results: while the k-means clustering method for picking and weighing representative days produces an error margin of $20 \%$ or less for greater than 50 time steps, the random picking of days is unstable for all number of time steps tested.

\section{Conclusions and Policy Implications}

Given the importance of VRES capacity investment to most industrialised countries' energy policies, power system modelling that provides a credible basis for devising appropriate regulation is desirable. Thus, large-scale models that rely only on demand-based time slicing are inadequate for assessing power systems with a substantial share of VRES capacity. Indeed, neglecting the dependence between demand and VRES output could bias a model's predicted VRES capacity share, thereby undermining the basis for policy analysis. In this paper, two time-reduction methods for representing VRES output are compared in order to determine their suitability for inclusion in policy-enabling power system planning models.

It is shown that the integral method for time reduction is well suited to represent variability in a one-node model. To predict VRES capacity well, it is enough to use 30-40 time steps if the time steps are picked using the k-means clustering method described in Nahmmacher et al. (2016) and Wogrin et al. (2014). The representative days method needs 160-200 time steps in order to predict VRES capacity with an accuracy of $10 \%$, which is the paper's main performance metric. The conclusions are that:

- For one-node models, at least when the penetration level is below the level where competition between variation management strategies may become important, the integral 
method seems to be suitable. The model then needs to be designed so that it be convenient to run with 30 time steps. This approach may be adopted in integrated assessment models (IAMs), for example.

- For network models, the representative days approach, with at least 20 such days (160 time steps), is recommended. The requirement of the time dimension exceeds many of the models used for, e.g., policy recommendations, which means that it may be necessary to simplify the models in some other aspect, such as technology description or spatial resolution.

- The preparation step pays off. It is important to apply a stringent method rather than to select periods at random or with some preconceived idea of representativeness.

For future work, the comparison of the two time-slicing methods could be extended to incorporate additional variation management tools, e.g., DSM, storage, and transmission. However, such a study would require enhancing the one-node model by adding constrained transmission lines with the requisite spatial representation. In this case, the representative days approach would have to be based on representative weeks in order to handle storage adequately, while the integral method would require transition matrices to account for both storage and transmission.

\section{References}

Conejo, A.J., L. Baringo, S.J. Kazempour, and A.S. Siddiqui (2016), Investment in Electricity Generation and Transmission: Decision Making under Uncertainty, Springer International, Switzerland.

Energy Information Administration (2017), Levelized Cost and Levelized Avoided Cost of New Generation Resources in the Annual Energy Outlook 2017, April 2017, Washington, D.C., U.S., https://www.eia.gov/outlooks/aeo/pdf/electricity_generation.pdf. 
European Commission (2011), Energy Roadmap 2050. Communication from the Commission to the European Parliament, the Council, the European Economic and Social Committee and the Committee of the Regions, COM(2011) 885 final, 15 December 2011, Brussels, Belgium, http://eur-lex.europa.eu/LexUriServ/LexUriServ.do?uri=COM: 2011:0885: FIN : EN : PDF.

European Commission (2015), Electricity and Heat Statistics, Eurostat, Brussels, Belgium, http://ec.europa.eu/eurostat/statistics-explained/index.php/Electricity_ and_heat_statistics\#Consumption_of_electricity_and_derived_heat.

Fraunhofer Institute for Solar Energy Systems (2013), Levelized Cost of Electricity Renewable Energy Technologies, November 2013, Freiburg, Germany, https://www.ise.fraunhofer.de/content/dam/ise/en/documents/publications/ studies/Fraunhofer-ISE_LCOE_Renewable_Energy_technologies.pdf.

Frew, B.A., S. Becker, M.J. Dvorak, G.B. Andresen, and M.Z. Jacobson (2016), "Flexibility Mechanisms and Pathways to a Highly Renewable U.S. Electricity Future," Energy 101: $65-78$.

Frew, B.A. and M.Z. Jacobson (2016), "Temporal and Spatial Tradeoffs in Power System Modeling with Assumptions About Storage: An Application of the POWER Model," Energy 117: 198-213.

Haller, M., S. Ludig, and N. Bauer (2012), "Decarbonization Scenarios for the EU and MENA Power System: Considering Spatial Distribution and Short Term Dynamics of Renewable Generation," Energy Policy 47: 282-290.

Lehtveer, M., N. Mattsson, F. Hedenus, and M. Soini (2016), "Using Resource Based Slicing to Capture the Intermittency of Variable Renewables," working paper, Department of Energy and Environment, Chalmers University of Technology, Gothenburg, Sweden, http: //publications.lib.chalmers.se/records/fulltext/238707/local_238707.pdf. 
Lund, H., P. A. Østergaard, D. Connolly, and B.V. Mathiesen (2017), "Smart Energy and Smart Energy Systems," Energy 137: 556-565.

Lund, H. and J.Z. Thellufsen (2017), "Advanced Energy Systems Analysis Computer Model: Documentation Version 13," Sustainable Energy Planning Research Group, Aalborg University, Aalborg, Denmark, http://www.energyplan.eu/wp-content/uploads/2017/ 11/documentation.pdf.

MacDonald, A.E., C.T.M. Clack, A. Alexander, A. Dunbar, J. Wilczak, and Y. Xie (2016), "Future Cost-Competitive Electricity Systems and Their Impact on U.S. $\mathrm{CO}_{2}$ Emissions," Nature Climate Change 6: 526-531.

Merrick, J.H. (2016), "On Representation of Temporal Variability in Electricity Capacity Planning Models," Energy Economics 59: 261-274.

Nahmmacher, P., E. Schmid, L. Hirth, and B. Knopf (2016), "Carpe Diem: A Novel Approach to Select Representative Days for Long-Term Power System Modeling," Energy 112: 430442.

Nahmmacher, P., E. Schmid, and B. Knopf (2014), Documentation of LIMES-EU - A Long-Term Electricity System Model for Europe, Potsdam Institute for Climate Impact Research, Potsdam, Germany, https://www.pik-potsdam.de/members/paulnah/ limes-eu-documentation-2014.pdf.

Odenberger, M., T. Unger, and F. Johnsson (2009), "Pathways for the North European Electricity Supply," Energy Policy 37(5): 1660-1677.

Pfenninger, S., A. Hawkes, and J. Keirstead (2014), "Energy Systems Modeling for TwentyFirst Century Energy Challenges," Renewable and Sustainable Energy Reviews 33: 74-86.

Poncelet, K., E. Delarue, D. Six, J. Duerinck, and W. Dhaeseleer (2016), "Impact of the Level of Temporal and Operational Detail in Energy-System Planning Models," Applied Energy 162: 631-643. 
Reichenberg, L., A. Wojciechowski, F. Hedenus, and F. Johnsson (2017), "Geographic Aggregation of Wind Power-An Optimization Methodology for Avoiding Low Outputs," Wind Energy 20(1): 19-32.

Short, W., P. Sullivan, T. Mai, M. Mowers, C. Uriarte, N. Blair, D. Heimiller, and A. Martinez (2011), Regional Energy Deployment System (ReEDS), NREL/TP-6A20-46534, National Renewable Energy Laboratory, Golden, CO, U.S., http://www.nrel.gov/docs/ fy12osti/46534.pdf.

Tejada-Arango, D.A., S. Wogrin, and E. Centeno (2017), "Representation of Storage Operations in Network-Constrained Optimization Models for Medium- and Long-Term Operation," IEEE Transactions on Power Systems, forthcoming.

Virasjoki, V., A.S. Siddiqui, B. Zakeri, and A. Salo (2018), "Market Power with Combined Heat and Power Production in the Nordic Energy System," IEEE Transactions on Power Systems, forthcoming.

Wogrin, S., P. Dueñas, A.R. Delgadillo, and J. Reneses (2014), "A New Approach to Model Load Levels in Electric Power Systems with High Renewable Penetration," IEEE Transactions on Power Systems 29(5): 2210-2218.

Wogrin, S., D. Galbally, and J. Reneses (2016), "Optimizing Storage Operations in Mediumand Long-Term Power System Models," IEEE Transactions on Power Systems 31(4): 3129-3138.

Zhao, B., A.J. Conejo, and R. Sioshansi (2018), "Coordinated Expansion Planning of Natural Gas and Electric Power Systems," IEEE Transactions on Power Systems 33(3): 3064-3075.

\section{Appendix: Analysis with VRES Overgeneration}

Following the approach of Frew et al. (2016), we VRES overgeneration (or VRES curtailment) is used as another metric for comparing time-reduction methods. Using this metric 
reinforces the point about the shortcomings of the demand-based integral method. This metric is calculated as follows:

1. Obtain the optimal VRES capacity for each time-reduction method from Figure 3.

2. Insert the optimal VRES capacity for each time-reduction method into the benchmark model, i.e., "full" representation of time, with operational decisions only.

3. Run the benchmark operational model, i.e., with "full" representation of time, and calculate VRES overgeneration (or VRES curtailment) of each time-reduction method.

Figure 8 plots the VRES overgeneration calculated in this manner for the German region, where the VRES overgeneration from the benchmark model is close to zero. The results are similar for other regions and largely confirm the findings in Figure 3, i.e., that regardless of the number of time steps, the demand-based integral method does not provide a precise indication of VRES penetration. In this case, because it insists on adopting a high VRES capacity, the demand-based integral method overgenerates VRES by $40 \%$ relative to the benchmark model. By contrast, the representative days approach exhibits a similar degree of VRES overgeneration as the demand-based integral method for only a single representative day and quickly improves its performance when the number of representative days is increased. 


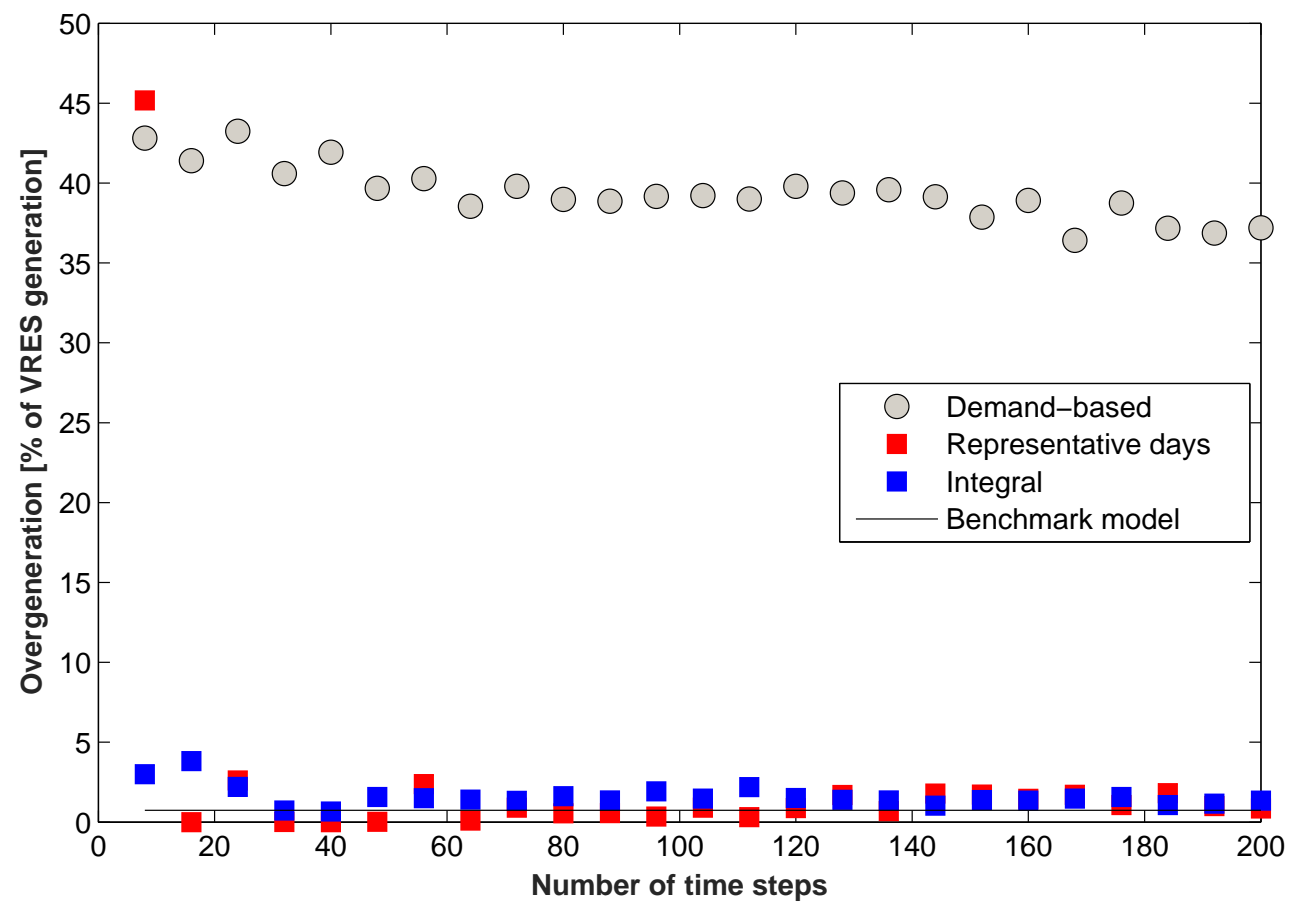

Figure 8: Implied VRES overgeneration under various time-reduction methods (German region) 